\title{
Hidden information in three-axial ECG data of normal subjects: Fractal dimensions of corresponding points from successive QRS loops as a potential sport and age dependent marker
}

\author{
Srdjan Čalošević ${ }^{1}$, Kristijan Dinjar ${ }^{1}$, Suzana Čalošević ${ }^{1}$, Sven Kurbel $^{2}$ and Robert Steiner ${ }^{2}$ \\ ${ }^{1}$ Osijek University Hospital Center, J. Huttlera 4, Osijek, Croatia \\ ${ }^{2}$ Osijek Medical Faculty, J. Huttlera 4, Osijek, Croatia
}

\begin{abstract}
This study is aimed to estimate spatial variability of normal QRS loops trajectories in successive cardiac cycles analyzed in XYZ phase space among 27 young and 27 older subjects, both male and female. Among young subjects, 10 individuals were professional football players, while the remaining 17 were students without regular physical activities. Modified three-axial leads (Frank's lead system) were used continually to record high-resolution ECG (1 kHz sampling rate) at rest during 200 seconds by Biopac Student Lab System. Variability of all the three spherical coordinates of the ventricular electrical vector in five characteristic positions regarding the $\mathrm{R}$ peak was analyzed. It was found that although fractal dimensions were not gender-dependent $(p>0.05)$, the calculated values in all tested five positions were greater in young than in older individuals $(p<0.05)$. Besides that, among young subjects, several fractal dimensions were greater in athletes than in physically inactive students $(p<0.05)$. These results suggest that the availability of possible phase space trajectories for QRS vector motion is related to individual physical activity and ageing, leaving less variability to physically inactive or older individuals. Recognizing noninvasive features of heart activity might help early detection of heart problems caused by aging and sedentary life style.
\end{abstract}

Key words: Electrocardiography - High resolution - Fractal dimension - QRS complex - Sport medicine - Ageing

\section{Introduction}

Contrary to a usual concept of homeostasis, many physiological systems, including a normal cardiac rhythm of a healthy heart, oscillate even in resting conditions. Electrical activity in the heart is so complex that it cannot be described as a simple rhythm, or frequency, with an increasing amount of evidence that the heart is not a periodic oscillator in normal physiological conditions (Goldberger et al. 1985a, 1985b, 2002; West et al. 1999; Sperelakis 2000; Paar et al. 2001; Sharma 2009; Losa 2014).

Assuming electrical activity of the heart is a chaotic dynamic system, the endpoint of a dominant electrocardiographic vector exhibits trajectories in the phase space that

Correspondence to: Sven Kurbel, Osijek Medical Faculty, J. Huttlera 4, 31000 Osijek, Croatia

E-mail: sven@jware.hr discretely vary from a cardiac cycle to a cardiac cycle, thus possibly showing characteristics of a strange attractor. It seems plausible that changes in consecutive QRS curve show fractal structure due to chaotic nature of electrical activity in the healthy heart.

Analysis of frequency in severe arrhythmia, such as ventricular fibrillation and torsades de pointes, reveals significant periodic patterns in the heart rhythm disturbances and shows that heart rhythm variability is significantly reduced during such disturbances. For example, there is a limited frequency range in ventricular fibrillation, which shows that there is much greater periodicity in generating impulses (Goldberger et al. 1985a, 1985b; Goldberger and West 1987; Mäkikallio et al. 1999).

It has been noted that illness and ageing diminish complexity of cardiac activity, which suggests that there is need to develop methods to quantify variability of cardiac activity. Common methods developed in the past decades 
have focused on analysis of R-R variability and it has been widely accepted that patients with preserved variability of $\mathrm{R}-\mathrm{R}$ intervals in ECG readings regulated by autonomous nervous system have better chances of survival (Task Force of the European Society of Cardiology (1996), Huikuri et al. 2003).

Normal electrical impulse in the heart, originating in sinoatrial node (SA node), propagates through conductive and muscle tissue as depolarization wave through the whole heart. This wave can be imagined as a series of single electric dipoles (having one positive and one negative end). These small single dipoles are oriented toward local wavefront and their total sum represents the total dipole of the heart muscle in that moment.

Electric dipole of the heart moves in the three-axial phase space. Detailed analysis of this motion is possible only using high frequency recording and three pairs of electrodes corresponding to X, Y and Z axes of the coordinate system (Frank's lead system and its modifications) (Frank 1954, 1956; Burch et al. 1953; Langner et al. 1958; Von Der Groeben 1967).

Beside anatomical structures of the conduction system, the depolarization wave path in the heart ventricles depends on the several factors: distribution of the heart muscle tissue within the thoracic cavity, speed of depolarization in small tissue volumes, telediastolic filling of ventricles determines the presystolic heart shape and its intrathoracic position, beside the dynamic of systolic blood ejection. Some of these factors are constitutional and thus stable, but others are variable and indirectly linked to the R-R variability that changes duration of heart filling. The remaining factors can be slowly adaptable (i.e., afterload and preload), while some can be expected to be nonlinear (changes due to respiration are out of sync with heart activity, the Frank Sterling mechanism changes each heart muscle contraction etc.). Noting that most of these linear and nonlinear factors are present during the QRS wave propagation is important.

Nonlinear (or chaotic) changes in QRS loop trajectories are expected to be detectable as increased variability between the corresponding measurements in consecutive loops. This variability of short QRS segments possibly depends on the number of available loop paths due to anatomical, physiological or functional constraints imposed on the electric wave spreading through the ventricular muscle.

The working hypothesis is that movements of depolarization waves in phase space are chaotically variable in successive cardiac cycles, making the corresponding positions along the consecutive QRS loops always close to each other, but never identical. In other words, variable shifts in trajectories of successive cardiac cycles are expected. A plausible extrapolation of this idea is that measurement of variability in spatial ECG data in healthy subjects might ensure alternative diagnostic approach to adaptive variability of cardiac activity in health and disease.
In addition, it should be here emphasized that analysis of variability of QRS loop trajectories in successive cardiac cycles is clearly distinguishable from the usual analysis of R-R variability focused on the variability of the heart pacemaker in time, while here proposed approach use phase space coordinates to test spatial variability of the QRS loop.

\section{Materials and Methods}

This study was designed to detect size effect of 0.7 at the level of significance of 0.05 with strength of 0.8 , so program $G^{\star}$ Power 3.1.2 (Franz Faul, Universität Kiel, Germany) calculated that at least 26 subjects should be included in each group of subjects used for comparison. The sample included 54 healthy subjects who volunteered for the research:

The first group of 27 older subjects included some volunteers and patients enlisted for surgery. Examinations were done by an internal medicine specialist and an anaesthesiologist to assess patients' cardiopulmonary status (anamnesis, blood pressure - RR method, hearth and lungs physical examination, ECG). Only individuals with normal findings were included.

The second group of 27 young subjects included 17 healthy students and 10 football players.

Arterial blood pressure was taken and physical examination of the heart and lungs was done. All subjects had a normal standard ECG. Young subjects were 21.52 \pm 2.75 years old on average, while older subjects were $53.41 \pm 5.66$ years old. There were more male subjects than female ( 34 and 20, respectively).

In the research lab every subject was placed three pairs of electrodes to obtain three-lead recordings (Frank's lead system) corresponding to three axes of the coordinate system (X axis - horizontal, $\mathrm{Y}$ axis - vertical, and $\mathrm{Z}$ axis - frontto-back) (Frank 1954, 1956). The $\mathrm{X}$ axis was directed from the right to the left axilla, the $\mathrm{Y}$ axis was parallel with the vertical body axis going through the center of the body, and $\mathrm{Z}$ axis was directed front-to-back through the thorax at the heart level.

After the placement of electrodes, subjects rested in lying position for five minutes before ECG. Continuous ECG recording was taken using Biopac Student Lab (http:// www.biopac.com/) over 200 seconds with a sampling rate of $1000 \mathrm{~Hz}$.

The obtained data represented voltage values in $\mathrm{X}, \mathrm{Y}$ and $Z$ direction of a three-axial phase coordinate system, i.e., voltage values of the depolarization wave in the phase space.

Detection of an R peak by the Biopac Student Lab consists of two phases. The first is deflection from the baseline, the second starts when the peak is passed and voltage starts to decrease. Then software recognizes that it was a peak. So, the 
$0^{\text {th }} \mathrm{ms}$ is delayed from the actual peak. Beside that, software uses one lead for detection, so the $3 \mathrm{D}$ vector can achieve even higher resultant potential (in $\mathrm{mV}$ ), due to other two leads, few milliseconds before or after the monitored lead. This delay of the $0^{\text {th }} \mathrm{ms}$ behind the maximal voltage is visible in Figures 1 and 2.

The obtained data were used to superpose the recorded cardiac cycles so that they coincided at the point where the $\mathrm{R}$ wave deflects downwards. Superposing enables accurate sampling of the phases in QRS complex: an original program detects the peak of the $\mathrm{R}$ amplitude and then separates $60 \mathrm{~ms}$ before and $59 \mathrm{~ms}$ after the peak. Thus, the data are filtered in time, so that $120 \mathrm{~ms}$ of QRS loop were isolated from every cardiac cycle being superposed in the R-wave peak millisecond.

Electric potentials were converted to put the center of the three-axial phase space coordinate system at a point representing arithmetic mean of measured voltages in each of the three axes (X, Y and $\mathrm{Z}$ ). Placing the observing point into the center of the QRS loop enabled a similar perspective in observation of all parts of the loop and thus observation of curve variability in successive cardiac cycles with minimum perspective distortion.

\section{Converting the three-axial ECG data into spherical coordinates}

Since a series of measured points behaves as a loop circling the center of a three-axial phase coordinate system, the voltages were converted into spherical coordinates: radius, latitude and longitude (Figure 1):

- radius is a distance (expressed in $\mathrm{mV}$ ) that connects the QRS loop trajectory in each millisecond with the center of the three-axial coordinate system. Among spherical coordinates, the radius corresponds to altitude from the center;

- latitude of each radius is the angle between that radius and the $\mathrm{XZ}$ plane. In spherical coordinates, the $\mathrm{XZ}$ plane is the equator, and latitude ranges from $-90^{\circ}$ to $+90^{\circ}$;

- longitude of each radius is the angle of that radius in the equatorial XZ plane. In spherical coordinates, the longitude ranges from $0^{\circ}$ to $+360^{\circ}: 0^{\circ}$ is the positive $\mathrm{X}$ axis directed at the left axilla, $90^{\circ}$ towards sternum, $180^{\circ}$ directed to the right axilla, $270^{\circ}$ directed straight to the back of thorax.

\section{Calculation of fractal dimensions}

Variability of superposed QRS loops in phase space was tested separately for radius $(\mathrm{mV})$, latitude (degrees) and longitude (degrees). The obtained data for specific indicators were presented in a table that had 120 columns in width (representing milliseconds) containing one QRS loop in each row. These milliseconds were deliberately chosen: the peak of the $\mathrm{R}$ wave was considered the $0^{\text {th }} \mathrm{ms},-20^{\text {th }},-15^{\text {th }}$ and $-10^{\text {th }} \mathrm{ms}$ before the downward deflection of the $\mathrm{R}$ amplitude and $+5^{\text {th }} \mathrm{ms}$ after the downward portion of the QRS loop (Figure 2).

Linear sequences of values for each of three polar coordinates in successive QRS loops were analyzed by calculating fractal dimension of links between corresponding points as a measure of path availability in the phase space.

Two methods for calculation of fractal dimensions for selected milliseconds were chosen from the program package TruSoft Int'l, Inc. Benoit (http://www.trusoft-international. $\mathrm{com} /$ ).

\section{$R / S$ (range/scale) analysis method}

Within an interval, or window, of length $w$ in a trace two quantities can be defined: $R(w)$, the range taken by the values of $y$ in the interval. $S(w)$, the standard deviation of the first differences delta $y(\mathrm{~d} y)$ of the values of $y$ within the window. The first differences of the $y$ axis are defined as the differences between the values of $y$ at some location $x$ and $y$ at the previous location on the $x$ axis:

$$
\mathrm{d} y(x)=y(x)-y(x-\mathrm{d} x)
$$

where delta $x(\mathrm{~d} x)$ is the sampling interval, i.e., the interval between two consecutive values of $x$.

A reliable measurement of $S(w)$ requires data with a constant sampling interval $\mathrm{d} x$, because the expected difference between successive values of $y$ is a function of the distance separating them. $S(w)$ in the rescaled range method is used to standardize the range $R(w)$ to allow comparisons of different data sets.

The rescaled range $R / S(w)$ is defined as:

$$
R / S(w)=\langle R(w) / S(w)>
$$

where $w$ is the window length and the angled brackets $<R(w)>$ denote the average of a number of values of $R(w)$. The basis of the method is that, because of self-affinity, one expects the range taken by the values of $y$ in a window of length $w$ to be proportional to the window length to a power equal to the Hurst exponent $H$, i.e.,

$$
R / S(w)=w^{H}
$$

In practice, for a given window length $w$, one subdivides the input series in a number of intervals of length $w$, measures $R(w)$ and $S(w)$ in each interval, and calculates $R / S(w)$ as the average ratio $R(w) / S(w)$. This process is repeated for a number of window lengths, and the logarithms of $R / S(w)$ are plotted versus the logarithms of $w$. If the trace is selfaffine, this plot should follow a straight line whose slope 

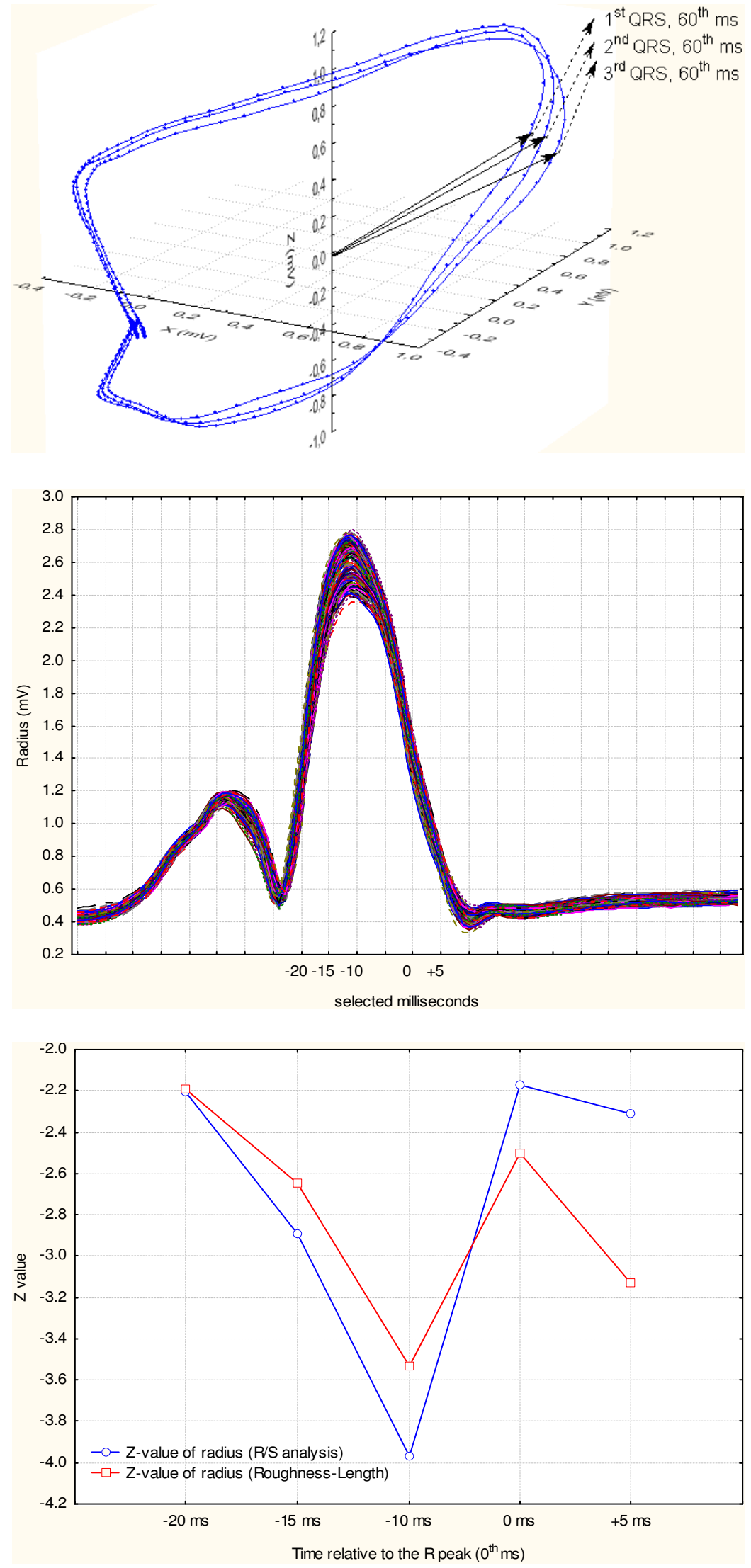

Figure 1. 3D view of three superposed QRS loops (during $120 \mathrm{~ms}$ ). Radius is a dart drawn from the centre of QRS loops, with latitude and longitude.

Figure 2. High resolution ECG recordings: $120 \mathrm{~ms}$ of QRS loop isolated from successive cardiac cycles, superposed by the moment of $\mathrm{R}$-wave peak detection by the Biopac Student Lab (shown as the point 0 ). Other selected milliseconds were: $-20^{\text {th }},-15^{\text {th }}$ and $-10^{\text {th }}$ before the downward deflection of the $\mathrm{R}$ amplitude and $+5^{\text {th }} \mathrm{ms}$ after the downward portion of the QRS loop.

Figure 3. Analyzing $\mathrm{Z}$ values of radius in both fractal dimensions variants ( $R / S$ and RoughnessLength methods) as indicator of significance of difference between young and middle-aged subjects, significance increases (in absolute values) in ascending portion of the QRS loop with maximum value at $-10^{\text {th }} \mathrm{ms}$, and then decreases towards initial values in the $0 \mathrm{~ms}$. 
equals the Hurst exponent $H$. The fractal dimension of the trace can then be calculated from the relationship between the Hurst exponent $H$ and the fractal dimension:

$$
D r s=2-H
$$

where Drs denotes the fractal dimension estimated from the rescaled range method.

\section{Roughness-Length relationship method}

In the Roughness-Length relationship method one takes the standard deviation, or root-mean-square (RMS) roughness of the data in windows of size $w$, rather than the vertical range. For a self-affine trace, the RMS roughness $s(w)$, (where $\mathrm{s}=$ standard deviation) measured in a window of size $w$ is related to the Hurst exponent as:

$$
s(w) \sim w^{H}
$$

For a given window length $w$, the input series is subdivided in a number of intervals of length $w$, the RMS roughness is calculated in each window after subtracting a local linear trend. The final estimate is taken to be $s(w)$, the average of the RMS roughness measured in each window. This process is repeated for a number of window lengths, and the logarithms of $\langle s(w)\rangle$ (where the angled brackets $<>$ denote the average of a number of values of $s(w))$ are plotted versus the logarithms of $w$. If the trace is self-affine, this plot should follow a straight line whose slope equals the Hurst exponent $H$. The fractal dimension of the trace can then be calculated from the relationship between the Hurst exponent $H$ and the fractal dimension $D r l$ :

$$
\operatorname{Drl}=2-H
$$

where $\operatorname{Drl}$ denotes the fractal dimension estimated from the RMS roughness.

\section{Statistical data analysis}

To calculate fractal dimensions of variability of spherical coordinates, program package TruSoft Int'l, Inc. (1997, 1999) Benoit, version 1.31 was used (www.trusoft.netmegs. com). In statistical analysis of the obtained data and creation of tables and graphs, statistical program packages STATISTICA (data analysis software system), version 7 (StatSoft, Inc. 2004) and SPSS for Windows 9.0.0 were used.

\section{Results}

Main characteristics for two groups of subjects: young and older subjects (average age 21.52 \pm 2.75 and $53.41 \pm 5.66$, respectively) are presented in Table 1 . Mann-Whitney $U$ test was used to compare the two groups of subjects according to their basic features. Besides being significantly older than the young subjects $(p<0.001)$, the older subjects also had greater body mass $(p<0.001)$ in comparison to the young subjects. Systolic and diastolic blood pressure (BP) values were normal in both groups of subjects (120/75 in young and $135 / 80$ in older subjects), which meets the inclusion criteria, but both BP values were significantly higher in older subjects $(p<0.001$ and $p<0.001$, respectively). There was no significant difference in body height. One third of the young subjects and $25.93 \%$ among older subjects smoked cigarettes.

In Table 2 it can be seen that fractal dimension values for the analyzed milliseconds according to gender were significantly different only for the radius in $-10^{\text {th }} \mathrm{ms}$ where average fractal dimension values were significantly higher in female subjects. By the Roughness-Length method, in women fractal dimension values of latitude in $-20^{\text {th }} \mathrm{ms}$, radius in $-15^{\text {th }}$ and in $-10^{\text {th }} \mathrm{ms}$ were statistically significantly higher.

Fractal dimension values (R/S analysis) for all three spherical coordinates in all five selected milliseconds were higher in young subjects. Moreover, fractal dimension values

\begin{tabular}{|c|c|c|c|c|c|}
\hline \multirow{2}{*}{ Parameters } & \multicolumn{2}{|c|}{ Young subjects $(n=27)$} & \multicolumn{2}{|c|}{ Older subjects $(n=27)$} & \multirow{2}{*}{$p$} \\
\hline & Median & Mean \pm SD & Median & Mean \pm SD & \\
\hline Age (years) & 21 & $21.52 \pm 2.75$ & 52 & $53.41 \pm 5.66$ & $<0.001$ \\
\hline Height $(\mathrm{cm})$ & 174 & $175.11 \pm 10.29$ & 177 & $175.04 \pm 9.03$ & 0.729 \\
\hline Weight (kg) & 67 & $70.3 \pm 14.09$ & 88 & $88.96 \pm 15.51$ & $<0.001$ \\
\hline Systolic arterial pressure (mmHg) & 120 & $119.07 \pm 7.21$ & 135 & $133.15 \pm 15.07$ & $<0.001$ \\
\hline Diastolic arterial pressure $(\mathrm{mmHg})$ & 75 & $74.63 \pm 5.71$ & 80 & $83.52 \pm 12.62$ & 0.003 \\
\hline Share of males & \multicolumn{2}{|c|}{$48.15 \%$} & \multicolumn{2}{|c|}{$77.78 \%$} & \\
\hline
\end{tabular}
for radius in all five analyzed milliseconds and for longitude

Table 1. Basic features of 54 healthy volunteered subjects

The differences were tested by Mann-Whitney test. 
Table 2. Comparison of fractal dimensions of linear sequence of values for all three spherical coordinates by R/S and Roughness-Length method according to gender

\begin{tabular}{|c|c|c|c|c|c|c|c|}
\hline \multirow{2}{*}{$\begin{array}{l}\text { Time relative to } \\
\text { the R peak (ms) }\end{array}$} & \multirow{2}{*}{$\begin{array}{c}\text { Spheric } \\
\text { coordinates }\end{array}$} & \multicolumn{3}{|c|}{$\mathrm{R} / \mathrm{S}$ method } & \multicolumn{3}{|c|}{ Roughness-Length method } \\
\hline & & Women & Men & $p$ & Women & Men & $p$ \\
\hline$-20^{\text {th }}$ & latitude & 1.948 & 1.917 & 0.654 & 1.959 & 1.903 & 0.025 \\
\hline$-15^{\text {th }}$ & radius & 2.017 & 1.978 & 0.255 & 2.016 & 1.968 & 0.046 \\
\hline$-10^{\text {th }}$ & radius & 2.035 & 1.976 & 0.036 & 2.027 & 1.975 & 0.030 \\
\hline
\end{tabular}

The differences were tested by Mann-Whitney test.

in $-15^{\text {th }} \mathrm{ms}$ were statistically significantly higher in young subjects (Table 3). Analysis of fractal dimension values carried out by the Roughness-Length method showed higher values for all three spherical coordinates in all five selected milliseconds in young subjects. Statistically significant difference was noticed in fractal dimension values of radius in all five analyzed milliseconds, of longitude in $-20^{\text {th }},-15^{\text {th }}$, $-10^{\text {th }}$ and $+5^{\text {th }} \mathrm{ms}$ and of latitude in $-20^{\text {th }} \mathrm{ms}$ in young subjects. The same Table 3 also shows that among young subjects, athletes have also shown significantly higher fractal dimension values by the R/S method for several selected milliseconds. It was unexpected that values obtained by the Roughness-Length method did not discriminate well between athletes and students.

Analyzing $\mathrm{Z}$ values of radius in both fractal dimensions variants (R/S and Roughness-Length methods) as indicator of significance of difference between young and older subjects, it appears that significance increases (in absolute values) in the ascending portion of the QRS loop with maxi- mum value at $-10^{\text {th }} \mathrm{ms}$, and then decreases towards initial values in the $0^{\text {th }} \mathrm{ms}$ (Figure 3 ).

It means that a certain portion of the QRS loop is more significant in distinguishing young subjects from older ones and it is the ascending portion of the $\mathrm{R}$ amplitude. It can be concluded that in this portion of the QRS loop variability of spatial motion of ventricular electrical vector shows the greatest difference between young and older subjects.

\section{Discussion}

Similarly to other fields, variability as a reflection of system's inclination to chaotic behavior was recognized late in electrocardiography, and when it was recognized it was understood as a part of pathology.

Up to a recent time, certain limitations in analyzing variability of cardiac activity were present: limitations in measuring instruments, limitations in recording time, im-

Table 3. Comparison of fractal dimensions of linear sequence of values for spherical coordinates in five selected milliseconds (carried out by R/S analysis and Roughness-Length method) between young subjects and the elderly, and also between athletes and students

\begin{tabular}{|c|c|c|c|c|c|c|c|c|c|c|}
\hline \multirow{2}{*}{$\begin{array}{l}\text { Time relative } \\
\text { to the } \mathrm{R} \text { peak } \\
\text { (ms) }\end{array}$} & \multirow{2}{*}{$\begin{array}{l}\text { Spheric } \\
\text { coordinates }\end{array}$} & \multicolumn{2}{|c|}{$\begin{array}{l}\text { Median of fractal } \\
\text { dimension by } R / S\end{array}$} & \multirow{2}{*}{$p$} & \multicolumn{2}{|c|}{$\begin{array}{l}\text { Median of fractal } \\
\text { dimension by } \\
\text { Roughness-Length }\end{array}$} & \multirow[b]{2}{*}{$p$} & \multicolumn{2}{|c|}{$\begin{array}{l}\text { Median of fractal } \\
\text { dimension by R/S }\end{array}$} & \multirow[b]{2}{*}{$p$} \\
\hline & & $\begin{array}{c}\text { Young } \\
\text { subjects } \\
(n=27)\end{array}$ & $\begin{array}{c}\text { Older } \\
\text { subjects } \\
(n=27)\end{array}$ & & $\begin{array}{c}\text { Young } \\
\text { subjects } \\
(n=27)\end{array}$ & $\begin{array}{c}\text { Older } \\
\text { subjects } \\
(n=27)\end{array}$ & & $\begin{array}{l}\text { Young } \\
\text { athletes } \\
(n=10)\end{array}$ & $\begin{array}{l}\text { Students } \\
(n=17)\end{array}$ & \\
\hline \multirow{3}{*}{$-20^{\text {th }}$} & Latitude & 1.953 & 1.891 & 0.088 & 1.979 & 1.899 & 0.012 & 1.982 & 1.948 & 0.192 \\
\hline & Longitude & 1.993 & 1.949 & 0.059 & 2.003 & 1.933 & 0.006 & 2.044 & 1.969 & 0.016 \\
\hline & Radius & 1.968 & 1.899 & 0.027 & 1.981 & 1.907 & 0.029 & 2.000 & 1.933 & 0.315 \\
\hline \multirow{2}{*}{$-15^{\text {th }}$} & Longitude & 2.037 & 1.967 & 0.008 & 2.033 & 1.969 & 0.001 & 2.058 & 1.992 & 0.008 \\
\hline & Radius & 2.014 & 1.943 & 0.004 & 2.020 & 1.947 & 0.008 & 2.049 & 1.992 & 0.021 \\
\hline \multirow{2}{*}{$-10^{\text {th }}$} & Longitude & 2.007 & 1.938 & 0.100 & 2.024 & 1.979 & 0.013 & 2.025 & 2.007 & 0.353 \\
\hline & Radius & 2.040 & 1.934 & $<0.001$ & 2.034 & 1.974 & $<0.001$ & 2.042 & 2.035 & 0.175 \\
\hline 0 & Radius & 2.015 & 1.968 & 0.030 & 2.017 & 1.983 & 0.012 & 2.049 & 1.980 & 0.366 \\
\hline \multirow{2}{*}{$+5^{\text {th }}$} & Longitude & 1.966 & 1.954 & 0.421 & 1.961 & 1.946 & 0.025 & 1.985 & 1.926 & 0.015 \\
\hline & Radius & 2.007 & 1.938 & 0.021 & 2.017 & 1.978 & $<0.001$ & 2.030 & 1.991 & 0.393 \\
\hline
\end{tabular}

The differences were tested by Mann-Whitney. (Only significant differences are shown). 
possibility to correctly postprocess analogously recorded signals. Because of this, initial approaches to analyze ECG signal regarding variability were changes in R-R intervals after a change in body position, e.g., from lying into a sitting position, or after Valsalva maneuver, by which no special attention was paid to longer recordings.

A new era of research in this field started with development of Holter ECG, computerized ECGs that can record ECG without time limits in a suitable time and voltage resolution, introduction of low-noise electrodes with silver, and modern analogue-to-digital converters as input circuits.

As people were already oriented toward R-R variability as a key feature that every clinician analyzing the ECG curve can recognize, the researchers also focused on analysis of this variability of ECG in time dimension expressed as duration of R-R intervals. This approach has several important advantages, one of them being the fact that R-R interval is an ECG feature with very little sensitivity to noise. In R-R interval analysis, ventricular premature beat, extrasystole, can cause problems. If it is not present and there is a sinus rhythm, despite the quality of ECG recording, possible noise and physical activity always noted as high frequency disturbance, $\mathrm{R}-\mathrm{R}$ intervals can be accurately recognized. This is the reason most of the literature on cardiac activity variability is focused on variability dependent on time.

Pikkujämsä et al. (1999) have shown in their study that $\mathrm{R}-\mathrm{R}$ interval dynamics undergoes significant changes from childhood to old age in healthy persons. All traditional measures of heart frequency variability decrease with age, as it has been proved with decrease in total variance and decrease in strength range at all frequencies. However, there are significant differences between different measures related to years of age: children, for example, despite lesser total variability of heart frequency, have complexity and fractal characteristics similar to those in young adults. Progressive loss of complexity (increased regularity and predictability) and decrease in total variability of heart frequency occur from the middle to old age. It needs to be emphasized that observed loss of complexity and changes in fractal characteristics that occur with ageing, does not correlate with decrease in total variability of heart frequency.

Age-related changes in variability of cardiac activity were analyzed in the study by Vuksanović and Gal (2005). In their analysis, fractal and spectral values of variability of cardiac activity were used. These parameters resulted from theory of deterministic chaos. The sample included 60 healthy subjects divided into four groups according to age. It was concluded that normal ageing correlates with decrease in variability of cardiac activity, which can be seen in greater regularity and lesser variability. Significant decrease in high frequency strength with ageing leads to conclusion that the main cause of these changes is reduced activity of parasympathicus in this area (Vuksanović and Gal 2005).
Beckers et al. (2006) studied influence of ageing on nonlinear measures of variability of heart activity and differences according to gender for these measures. They compared ECG recordings for 135 women and 141 men between the ages of 18 and 71 years obtained by Holter ECG. It was concluded that all measures significantly correlated with age, showing constant and consistent decrease in fractal dimension and approximate entropy after the age of 40 . Circadian rhythm was noted in all parameters, except correlation dimension in women, which showed no circadian changes. Values of nonlinear measures of heart activity variability decrease with age. This may meet the idea of decrease in autonomous modulation of the heart rhythm with age. Men and women had equal values in nonlinear measures of variability of heart activity.

In research described here, motion of QRS loop depolarization vectors in phase space measurable in voltage units $(\mathrm{mV})$ was analyzed. Presented indicators of variability of QRS loop trajectories in successive cardiac cycles in selected milliseconds can significantly distinguish recordings in young subjects from those in older subjects. Thus, the age-dependent feature of QRS loop motion in phase space is confirmed. Different interpretations of this finding are possible, starting with changes in cardiac muscle structure, especially in the conduction system in interventricular septum, to decreased heart wall movements due to decreased heart compliance.

Described approach was not found in available literature, and the most similar research was analysis of stroke volume variation. This method was also not directly focused on time component ( $\mathrm{R}-\mathrm{R}$ interval), but on measuring volume changes as accurately as possible (expressed absolutely in $\mathrm{ml}$ or relatively in percentage of ejection fraction). Similarly, Liu et al. simultaneously analyzed variability of cardiac activity and variability of stroke volumes in a series of cardiac cycles and concluded that variability of stroke volumes ensures different information on autonomous nervous system activity, unlike variability of intervals between successive cardiac cycles. The research included 12 healthy men who volunteered for ECG recording, which was taken in standing position (Liu et al. 2004). Similarly, Mohan et al. (2012) developed a device working on the principle of photoplethysmography that very accurately determines nonlinear parameters of variability of cardiac activity. Infrared probes are used to measure changes in capillaries in the limbs that occur because of cardiac rhythm.

Several points need to be taken into consideration when interpreting the obtained results regarding age-related variability of depolarization front movements in phase space:

- normal, resting respiration, which has little influence on anatomical relations. Men dominantly breathe by diaphragm, while women use intercostal muscles and dia- 
phragm in 50:50 ratio. Breathing in and out slightly moves the electrode on the chest, which may result in recording a slightly different voltage by this electrode and thus shifting the subsequent QRS loop in the same millisecond into a slightly different position in phase space. If this was the most important reason for variability of spatial movement of the QRS loop, it would follow that variability did not depend on age, but did depend on gender. Since most men breathe from diaphragm and most women employ intercostal muscle when breathing, if respiration was the reason for variability of spatial movement of the QRS loop due to movement of electrodes, then variability should be higher in women;

- change in blood volume in the chest caused by negative pressure during respiration changes the flow of blood into the heart through venae cavae, thus changing volume loading of the heart, which causes increase/decrease of the heart rhythm. If this was the reason for variability of depolarization vector movements, great difference should not be according to age, and according to gender, since changes in chest volume between single breaths at rest are similar in men and women;

- it is possible that variability depends on age-related redundancy of electrical paths in the conduction system of the heart. If existence of spare routes ensures reliability in cardiac activity, especially in young subjects, noted variability of recorded trajectories might be evidence for this. However, precise explanation cannot be given without more complex research that would include parallel measurements of systolic and diastolic function of the heart.

The last, but not the least is the question what can be said if we apply this approach to cardiac patients. Among our older patients with normal conventional ECG recordings was a 57-year-old hypertonic male with a previously detected LV hypertrophy. He had R/S fractal dimension of 1.835 and Roughness-Length dimension of 1.735 . These values were lower than the median values of both groups (medians of young adults were for R/S 2.040 and for Roughness-Length 2.034, while older adults showed medians of 1.934 and 1.974, respectively). This can be used as an illustration that further study should be attempted particularly in hypertensive patients.

A separate question is whether this method can be used in patients with bundle branch blocks (BBB) that widen and deform QRS complex beyond $100 \mathrm{~ms}$. Technically it seems that there should be no difficulties since Biopac software can detect the first portion of the QRS complex by sensing the deflection peak and initial return toward the baseline. The open question is whether fractal dimensions of the deformed QRS due to the left or to the right $\mathrm{BBB}$ contains same type and quantity of information as can be found in normal QRS complexes.

\section{Conclusions}

It can be concluded that in the myocardium of our younger subjects, there are more possible trajectories for QRS vector movements in space. This is further evidence in favor of the thesis that spatial variability of the heart's electrical activity, along with already proved temporal variability, is more pronounced in young persons. The greatest difference in fractal dimensions of variability of spherical coordinates depending on subject's age has been found for distance from the center of the QRS loop (radius) in $-10^{\text {th }} \mathrm{ms}$ before the beginning of downward deflection of $\mathrm{R}$ amplitude.

Results of this study inspired a theoretic model of heart electric activity that describes P, QRS and T waves as unbalanced transitions between stable configurations of the heart electric field during P-R, S-T and T-P segments (Kurbel 2014).

It needs to be noted that standard ECG in normal adult subjects cannot determine the subject's age. In the presented study, a potential numerical age-dependent parameter is identified and our results suggest that higher numerical values of the fractal dimension of the vector path depolarization within the heart phase space are found in young healthy adults. Besides that, fractal dimension values found in young subjects seem related to the level of their physical activity.

Acknowledgement. This study was financed through grant 2192192382-2386 from Croatian Ministry of Science, Education and Sport.

Conflicts of interest. The authors have no conflict of interest to declare.

\section{References}

Beckers F., Verheyden B., Aubert A. E. (2006): Aging and nonlinear heart rate control in a healthy population. Am. J. Physiol. Heart Circ. Physiol. 290, H2560-2570

http://dx.doi.org/10.1152/ajpheart.00903.2005

Burch G. E., Abildskov J. A., Cronvich J. A. (1953): Vectorcardiography. Circulation 8, 605-613

http://dx.doi.org/10.1161/01.CIR.8.4.605

Frank E. (1954): A direct experimental study of three systems of spatial vectorcardiography. Circulation 10, 101-113 http://dx.doi.org/10.1161/01.CIR.10.1.101

Frank E. (1956): An accurate, clinically practical system for spatial vectorcardiography. Circulation 13, 737-749 http://dx.doi.org/10.1161/01.CIR.13.5.737

Goldberger A. L., Bhargava V., West B. J., Mandell A. J. (1985a): Nonlinear dynamics of the heartbeat: II. Subharmonic bifurcations of the cardiac interbeat interval in sinus node disease. Physica D: Nonlinear Phenomena 17, 207-214 
http://dx.doi.org/10.1016/0167-2789(85)90005-3

Goldberger A. L., Bhargava V., West B. J., Mandell A. J. (1985b):

On a mechanism of cardiac electrical stability. The fractal hypothesis. Biophys. J. 48, 525 http://dx.doi.org/10.1016/S0006-3495(85)83808-X

Goldberger A. L., West B. J. (1987a): Applications of nonlinear dynamics to clinical cardiology. Ann. N. Y. Acad. Sci. 504, 195-213 http://dx.doi.org/10.1111/j.1749-6632.1987.tb48733.x

Goldberger A. L., West B. J. (1987b): Fractals in physiology and medicine. Yale J. Biol. Med. 60, 421

Goldberger A. L., Amaral L. A., Hausdorff J. M., Ivanov P. C., Peng C. K., Stanley H. E. (2002): Fractal dynamics in physiology: alterations with disease and aging. Proc. Nat. Acad. Sci. USA 99 (suppl. 1), 2466-2472 http://dx.doi.org/10.1073/pnas.012579499

Huikuri H. V., Mäkikallio T. H., Perkiömäki J. (2003): Measurement of heart rate variability by methods based on nonlinear dynamics. J. Electrocardiol. 36, 95-99 http://dx.doi.org/10.1016/j.jelectrocard.2003.09.021

Kurbel S. (2014): A vector-free ECG interpretation with P, QRS \& T waves as unbalanced transitions between stable configurations of the heart electric field during PR, ST \& TP segments. Theor. Biol. Med. Model. 11, 10 http://dx.doi.org/10.1186/1742-4682-11-10

Langner P. H., Okada R. H., Moore S. R., Fies H. L. (1958): Comparison of four orthogonal systems of vectorcardiography. Circulation 17, 46-54 http://dx.doi.org/10.1161/01.CIR.17.1.46

Liu H., Yambe T., Sasada H., Nanka S., Tanaka A., Nagatomi R., Nitta S. I. (2004): Comparison of heart rate variability and stroke volume variability. Auton. Neurosci. 116, 69-75 http://dx.doi.org/10.1016/j.autneu.2004.09.003

Losa G. A. (2014): The fractal geometry of life. Riv. Biol. 102, 29-59

Mäkikallio T. H., Koistinen J., Jordaens L., Tulppo M. P., Wood N., Golosarsky B., Peng C. K., Goldberger A. L., Huikuri H. V. (1999): Heart rate dynamics before spontaneous onset of ventricular fibrillation in patients with healed myocardial infarcts. Am. J. Cardiol. 83, 880-884 http://dx.doi.org/10.1016/S0002-9149(98)01068-6
Mohan A., James F., Fazil S., Joseph P. K. (2012): Design and development of a heart rate variability analyzer. J. Med. Syst. 36, 1365-1371 http://dx.doi.org/10.1007/s10916-010-9597-6

Paar V., Pavin N., Rosandić M. (2001): Link between truncated fractals and coupled oscillators in biological systems. J. Theor. Biol. 212, 47-56 http://dx.doi.org/10.1006/jtbi.2001.2334

Pikkujämsä S. M., Mäkikallio T. H., Sourander L. B., Räihä I. J., Puukka P., Skyttä J., Peng C. K., Goldberger A. L., Huikuri H. V. (1999): Cardiac interbeat interval dynamics from childhood to senescence comparison of conventional and new measures based on fractals and chaos theory. Circulation 100, 393-399 http://dx.doi.org/10.1161/01.CIR.100.4.393

Sharma V. (2009): Deterministic chaos and fractal complexity in the dynamics of cardiovascular behavior: perspectives on a new frontier. Open Cardiovasc. Med. J. 3, 110-123 http://dx.doi.org/10.2174/1874192400903010110

Sperelakis N. (2000): Heart Physiology and Pathophysiology. (Eds. Y. Kurachi, A. Terzic, M. V. Cohen), Academic Press. p. $99-132$

Task Force of the European Society of Cardiology (1996): Heart rate variability standards of measurement, physiological interpretation, and clinical use. Eur. Heart J. 17, 354-381

http://dx.doi.org/10.1093/oxfordjournals.eurheartj.a014868

Von Der Groeben J. (1967): Decision rules in electrocardiography and vectorcardiography. Circulation 36, 136-147 http://dx.doi.org/10.1161/01.CIR.36.1.136

Vuksanović V., Gal V. (2005): Analysis of heart rhythm variability by linear and non-linear dynamics methods. Vojnosanit. Pregl. 62, 621-627 (in Serbian) http://dx.doi.org/10.2298/VSP0509621V

West B. J., Zhang R., Sanders A. W., Miniyar S., Zuckerman J. H., Levine B. D. (1999): Fractal fluctuations in cardiac time series. Physica A 270, 552-566 http://dx.doi.org/10.1016/S0378-4371(99)00175-2

Received: November 15, 2015

Final version accepted: February 10, 2016

First published online: July 22, 2016 